\title{
ATLAS Monte Carlo generator tunes to LHC data
}

\author{
Andy Buckley \\ University of Edinburgh \\ for the ATLAS Collaboration
}

IEEE Nuclear Science Symposium, Knoxville, 2010-11-01
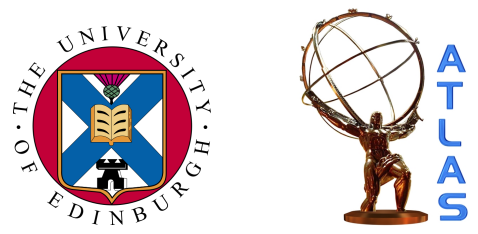


\section{The ATLAS detector}

General purpose detector designed to cover all the expected physics channels at LHC using $\mathrm{p}-\mathrm{p}$ collisions with energies up to $14 \mathrm{TeV}$ and $\mathrm{Pb}-\mathrm{Pb}$ collisions at $5.5 \mathrm{TeV} /$ nucleon.

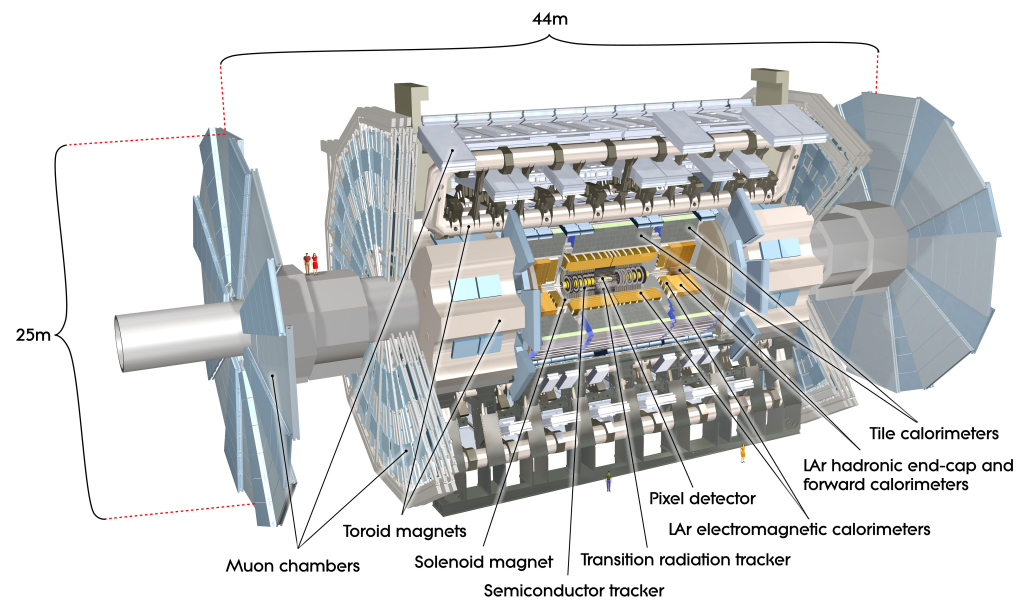




\section{MC modelling and tunes}

MC event modelling needed both for experimental understanding of QCD, $V+$ jets, etc. as backgrounds to new physics, and to allow highest-tech theory/data comparisons.

Some parts of MC simulation are very predictive - ME (especially at NLO), parton shower, QED, etc. - while those based on non-perturbative QCD are less robust. Hadronisation, "underlying event" in particular. These have parameters to be tuned to data.

Event simulation has two roles: to use and test our ability to calculate QCD, and to parameterise observables. Both are useful, but we need to be careful about which we are doing. Don't "tune away" failures in predictive parts. 


\section{MC generators in ATLAS}

ATLAS uses a multitude of event generator codes, in the appropriate scenarios:

- General purpose: PYTHIA, HERWIG, Pythia 8, Sherpa, Herwig++

- Multi-jet QCD: AlpGen, Sherpa, MadEvent

- NLO (in $\alpha_{s}$ ): MC@NLO, POWHEG, Herwig++

- Soft QCD: PHOJET, PYTHIA, HERWIG+JIMMY, Herwig++

- Other: HIJING, BlackMax, Charybdis, Protos, ExHuME, HORACE...

Initial interest is in tuning general purpose QCD to fit minimum bias QCD and underlying event ATLAS observables. For optimal simulation of QCD pile-up and signal-process UE respectively. 


\section{Tuning infrastructure}

PYTHIA and HERWIG+JIMMY are main ATLAS QCD

workhorse generators - for now. Most experience in tuning PYTHIA, which has many params, HERWIG+JIMMY is more restrictive.

Pre-LHC tuning rounds: MC08 and MC09. Mostly-manual tunes, based on CDF UE and min bias data. MC09 significantly changed from MC08, including change of PDF from CTEQ6L1 to MRST LO*. PYTHIA MC09c tune used Professor tool to improve colour-reconnection - since then all ATLAS tuning has been based on the Rivet/Professor tool combination (see previous talk).

Infrastructure also exists to use fast detector simulation to tune to pre-unfolding data - in practice not required as detector understanding has been better than anticipated. 


\section{Tuning data}

- Minimum bias from CDF and ATLAS: defined for ATLAS by 1 MBTS hit on each side. Diffractive contribution reduced via $N_{\mathrm{ch}} \geq 6$ requirement

- Underlying event from CDF 1 \& 2 with track-jet, calo-jet, and $Z \rightarrow$ leptons alignment

- Underlying event from ATLAS with leading track alignment, min bias trigger

- $\sqrt{s}=900 \mathrm{GeV} \& 7 \mathrm{TeV}$, track $p_{\perp}>500 \mathrm{MeV},|\eta|<2.5$ for all ATLAS results

leading jet/track/Z

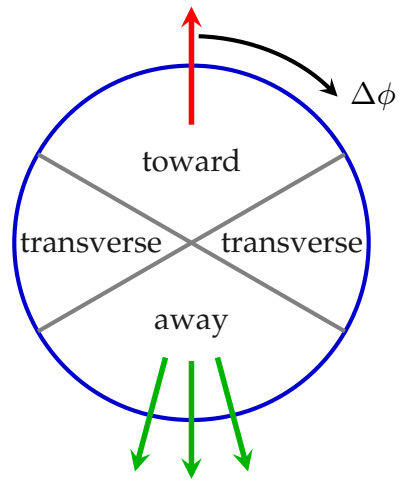

Underlying event analysis topology 


\section{PYTHIA AMBT1}

First LHC-data tune of PYTHIA was from ATLAS: AMBT1 (ATLAS Minimum Bias Tune \#1).

Focus on ATLAS min bias data, also including CDF min bias results. Test stability of pre-LHC energy evolution fit of multiple parton interactions (MPI) cutoff scale:

$$
p_{\perp}^{\min }=\operatorname{PARP}(82)\left(\frac{\sqrt{s}}{1.8 \mathrm{TeV}}\right)^{\operatorname{PARP}(90)}
$$

Other parameters: strength of colour reconnection (and suppression of high-momentum CR) and hadronic matter distribution (double-Gaussian).

Data used:

- diffractive-reduced min bias data with $N_{\mathrm{ch}} \geq 6$

- low-stats ATLAS UE

- plus pre-LHC MB/UE data from CDF Runs I \& II 


\section{PYTHIA AMBT1 params}

\begin{tabular}{llrr}
\hline Parameter & Meaning & MC09c & AMBT1 \\
\hline PARP(62) & ISR cutoff $(\mathrm{GeV})$ & 1.0 & 1.025 \\
PARP(93) & $k_{\perp}^{\text {prim }}$ cutoff $(\mathrm{GeV})$ & 5.0 & 10.0 \\
PARP(77) & Fast-string CR suppression & 0.0 & 1.016 \\
PARP(78) & CR strength & 0.224 & 0.538 \\
PARP(82) & $p_{\perp}^{\text {min }}$ & 2.31 & 2.292 \\
PARP(83) & Inner matter fraction & 0.8 & 0.356 \\
PARP(84) & Inner matter rel. width & 0.7 & 0.651 \\
PARP(90) & Energy evolution exp. & 0.24 & 0.250 \\
\hline
\end{tabular}

$p_{\perp}^{\min }$ and energy evolution as for MC09(c) - compatible with Tevatron. Matter distribution: first tune since MC08 so change expected. Non-zero fast-string CR suppression preferred. 


\section{AMBT1 tune constraints}
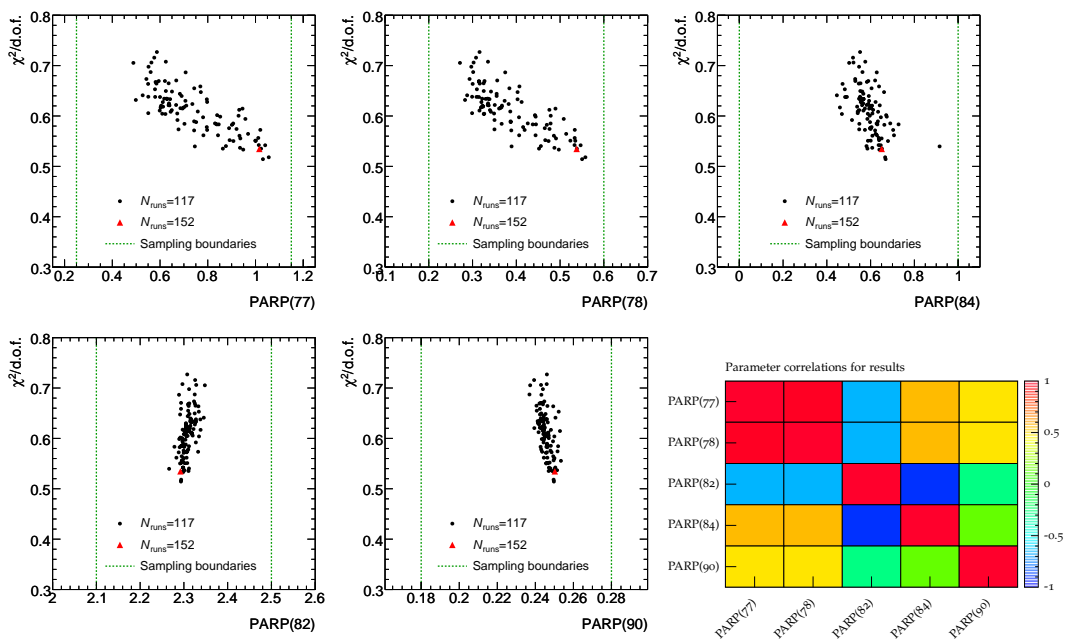


\section{PYTHIA tunes vs. ATLAS min-bias data $\left(N_{\mathrm{ch}} \geq 6\right)$ At $900 \mathrm{GeV}$}
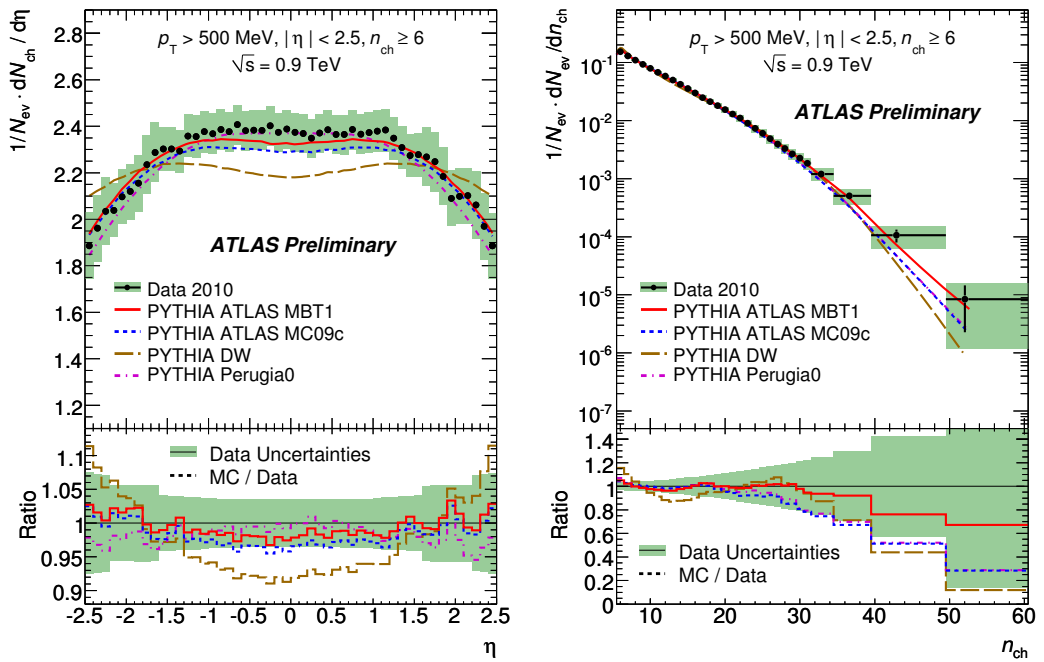


\section{PYTHIA tunes vs. ATLAS min-bias data $\left(N_{\mathrm{ch}} \geq 6\right)$ At $900 \mathrm{GeV}$}
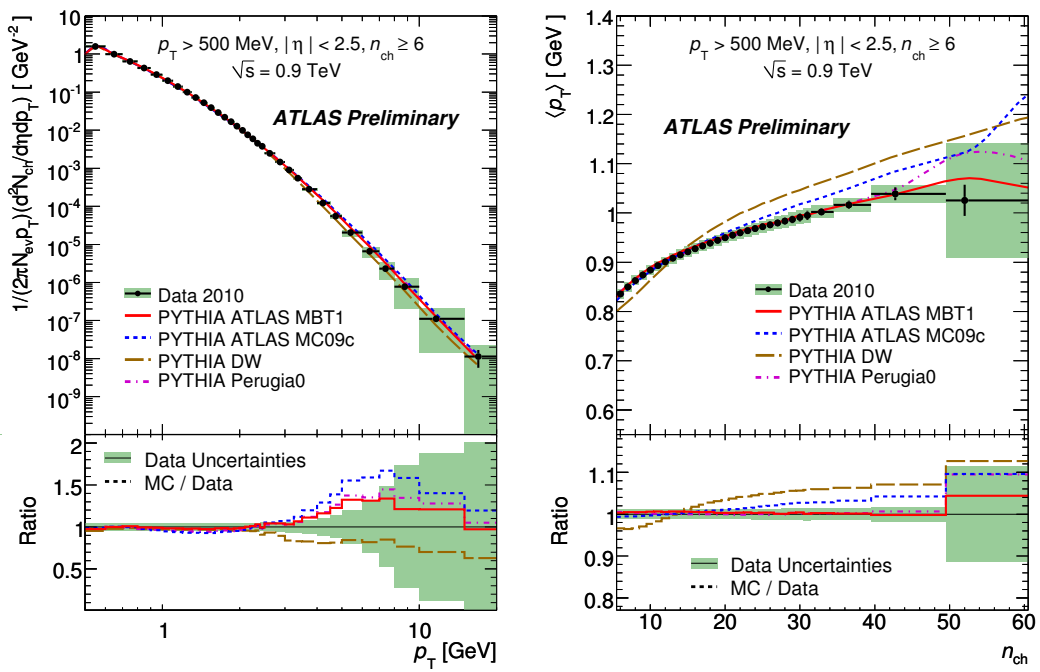


\section{PYTHIA tunes vs. ATLAS min-bias data $\left(N_{\mathrm{ch}} \geq 6\right)$}

\section{At $7 \mathrm{TeV}$}
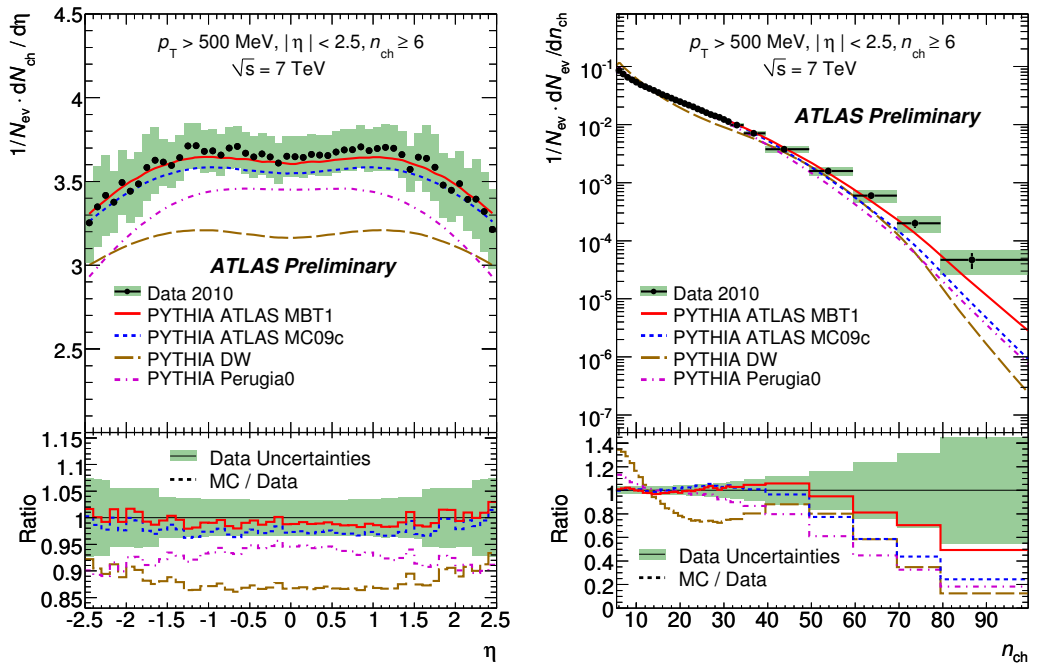


\section{PYTHIA tunes vs. ATLAS min-bias data $\left(N_{\mathrm{ch}} \geq 6\right)$}

\section{At $7 \mathrm{TeV}$}
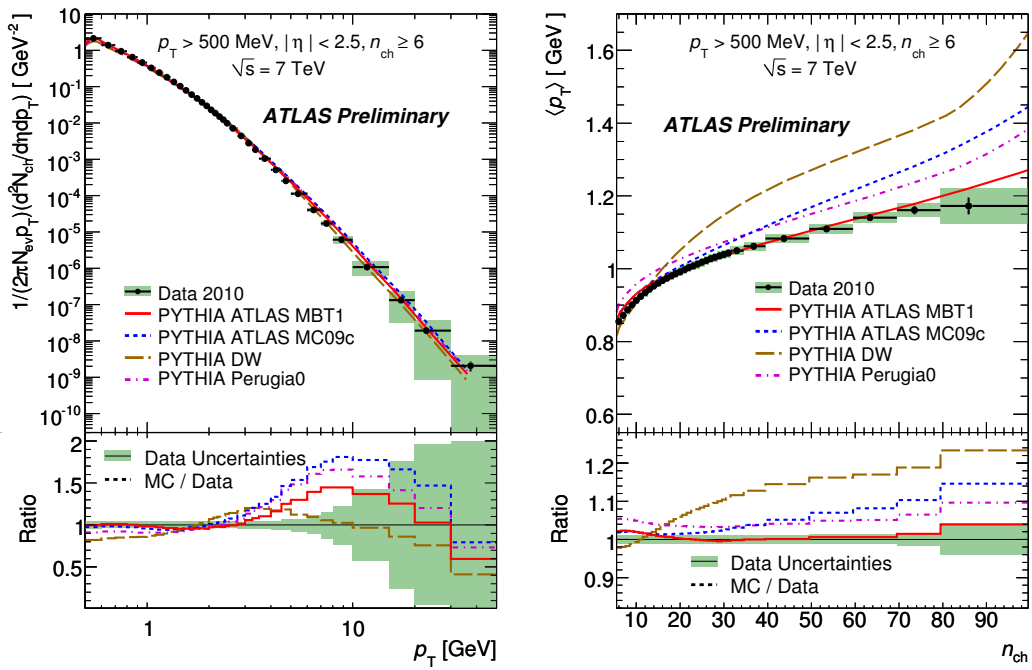


\section{PYTHIA tunes vs. ATLAS underlying event data}
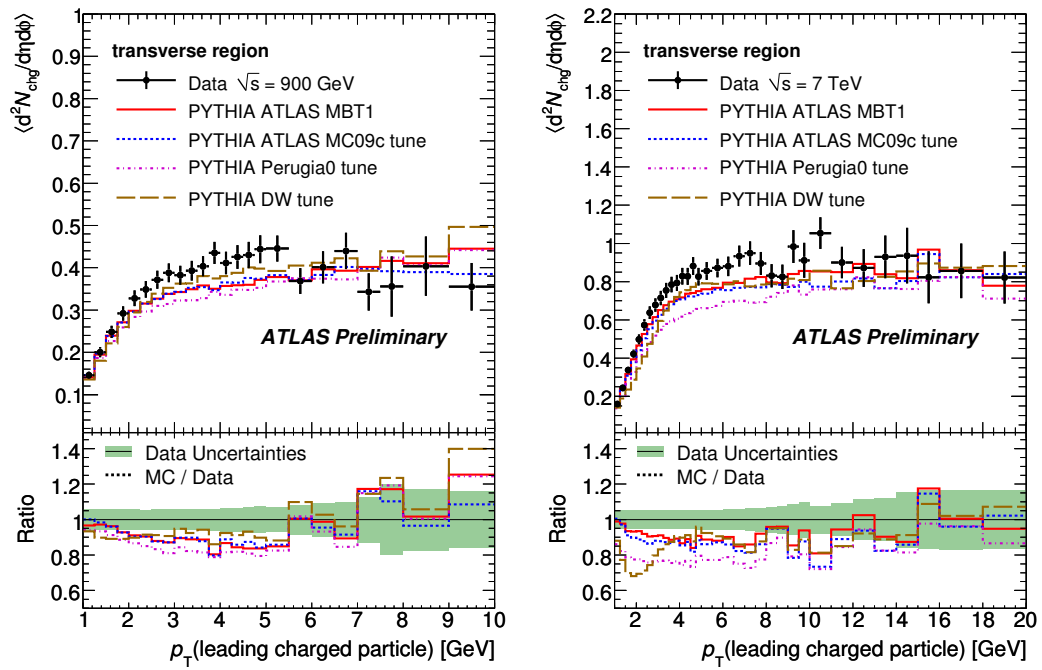


\section{PYTHIA tunes vs. ATLAS underlying event data}
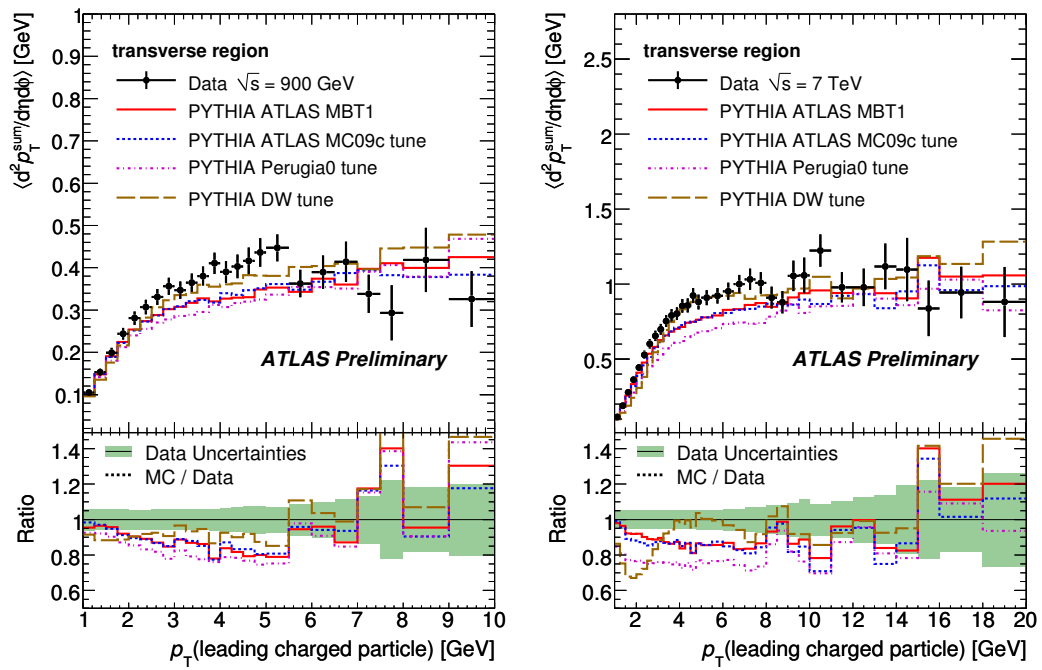


\section{HERWIG+JIMMY AUET1}

Main MC09 JIMMY tune was just an update of the manual MC08 tune from the CTEQ6L to MRST LO* PDF. Also a CTEQ6.6 NLO PDF tune using Professor. $\Rightarrow$ an updated tune similar to AMBT1 required.

BUT... JIMMY model only valid for secondary scattering in the presence of a hard interaction: can do underlying event but not min bias.

Hence a separate tune type for HERWIG+JIMMY: focus solely on UE data from CDF and ATLAS $\Rightarrow$ "AUET1" tune. 


\section{HERWIG+JIMMY AUET1 (ctd.)}

Fitting weights strictly ordered: ATLAS $>\mathrm{CDF}, 7 \mathrm{TeV}>$ $900 \mathrm{GeV}$, transverse $>$ toward/away. Also, $p_{\perp}^{\text {sum }}>N_{\mathrm{ch}}$ observable: JIMMY has no colour reconnection model (and hadronisation details not included in the tune.)

Use manual version of PYTHIA energy evolution ansatz:

$$
\text { PTJIM }=\text { PTMIN0 }\left(\frac{\sqrt{s}}{1.8 \mathrm{TeV}}\right)^{\text {EXP }}
$$

Tune PTMIN0 and EXP, plus hadronic matter distribution width PRRAD. Other params tweaked manually, particularly PTRMS (primordial $k_{\perp}$ ). 3 PDFs: LO*, CTEQ6L \& CTEQ66. 


\section{HERWIG+JIMMY AUET1 params}

\begin{tabular}{llrrrr}
\hline & & MC09 & & AUET1 & - \\
Parameter & Meaning & LO* & LO* & CTEQ6L1 & CTEQ6.6 \\
\hline \multicolumn{2}{l}{ Parameters fixed before numerical tuning } & & & & \\
ISPAC & ISR scheme & 0 & 2 & 2 & 2 \\
PTRMS & Prim. $k_{\perp}(\mathrm{GeV})$ & 0 & 1.2 & 1.2 & 1.2 \\
QSPAC & ISR cut-off $(\mathrm{GeV} / c)$ & 2.5 & 2.5 & 2.5 & 2.5 \\
\hline Tuned cutoff & meta-parameters & & & & \\
PTJIM0 & MPI cut-off $(\mathrm{GeV} / c)$ & 3.6 & 2.86 & 2.65 & 2.32 \\
EXP & MPI evolution & 0.274 & 0.273 & 0.277 & 0.220 \\
\hline Tuned JIMMY parameters & & & & \\
PRRAD & Proton radius $\left(\mathrm{GeV}{ }^{2}\right)$ & 2.2 & 1.69 & 1.90 & 1.82 \\
PTJIM(7) & MPI cut-off $(7 \mathrm{TeV})$ & 5.22 & 4.14 & 3.86 & 3.13 \\
PTJIM(10) & MPI cut-off $(10 \mathrm{TeV})$ & 5.76 & 4.56 & 4.26 & 3.38 \\
PTJIM(14) & MPI cut-off $(14 \mathrm{TeV})$ & 6.32 & 5.00 & 4.68 & 3.64 \\
\hline
\end{tabular}

Again mostly stable $p_{\perp}^{\min }$ and energy evolution. Note that MPI cut-off is ordered by magnitude of gluon PDF at low- $x$. 


\section{AUET1 tune constraints (for LO $*$ PDF only)}
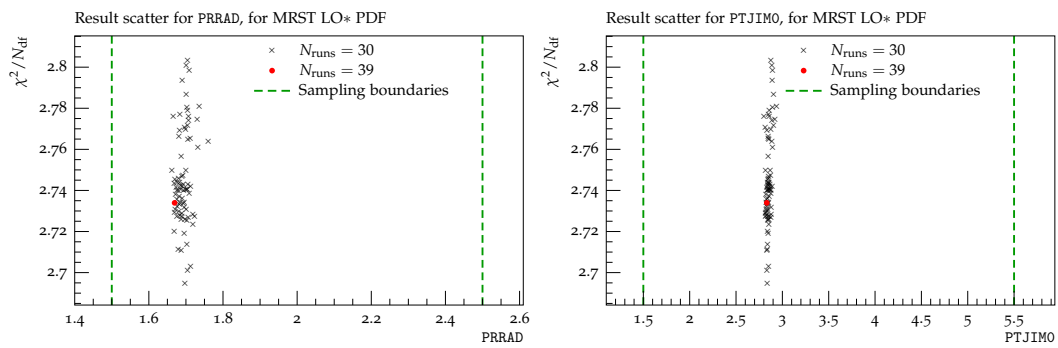

Result scatter for EXP, for MRST LO* PDF
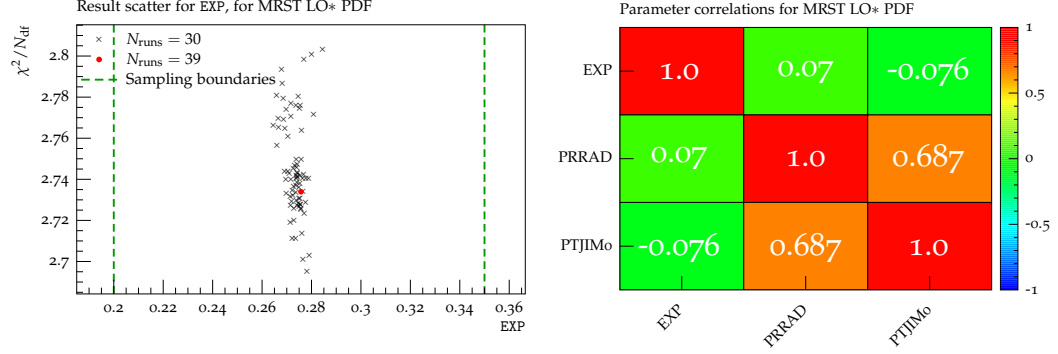


\section{AUET1 vs. CDF underlying event data}

Showing low- $p_{\perp}$ track jet UE at $1800 \mathrm{GeV}$ only: others in fit

\section{Transverse $N_{\mathrm{ch}}$}

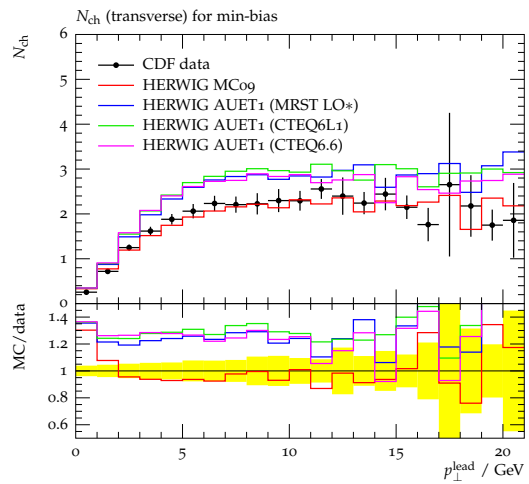

Transverse $p_{\perp}^{\text {sum }}$

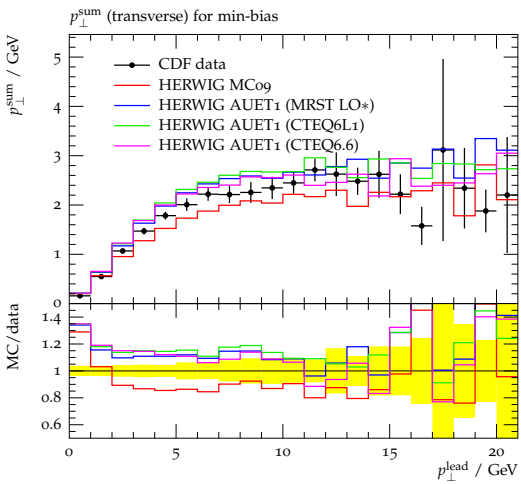




\section{AUET1 vs. ATLAS underlying event data}

\section{$900 \mathrm{GeV}$}

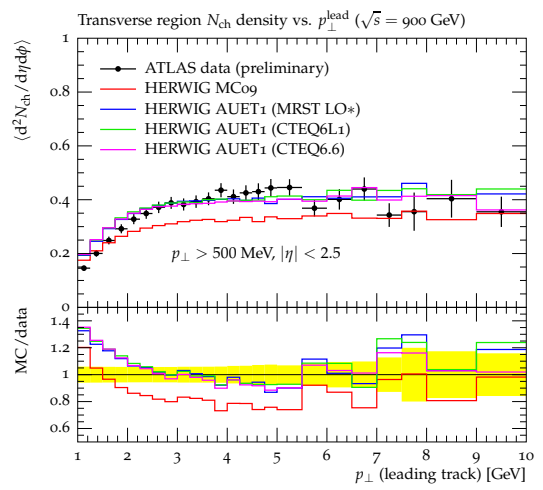

\section{$7 \mathrm{TeV}$}

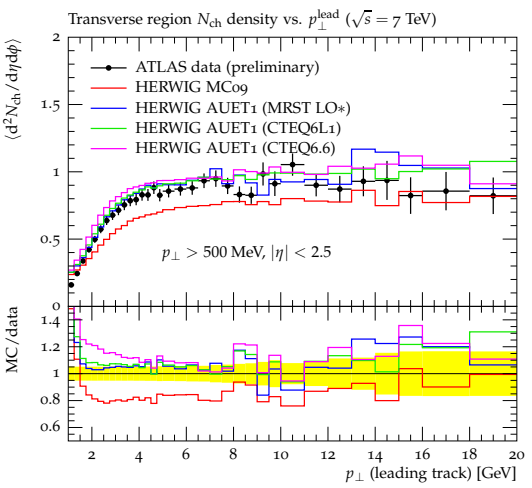




\section{AUET1 vs. ATLAS underlying event data}

\section{$900 \mathrm{GeV}$}

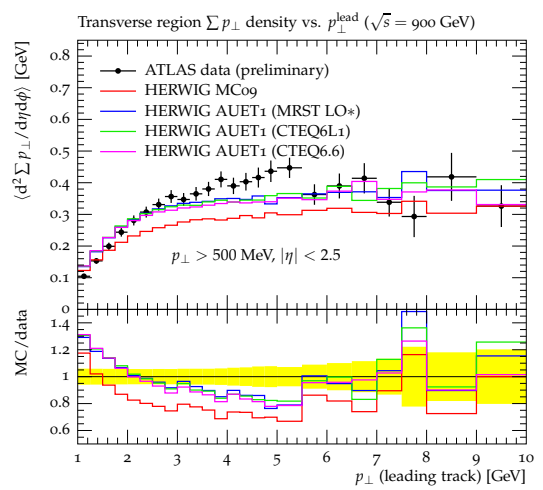

\section{$7 \mathrm{TeV}$}

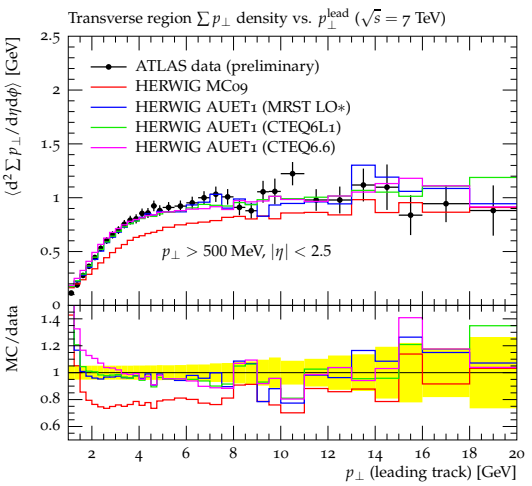




\section{AUET1 vs. ATLAS underlying event data}

\section{$900 \mathrm{GeV}$}

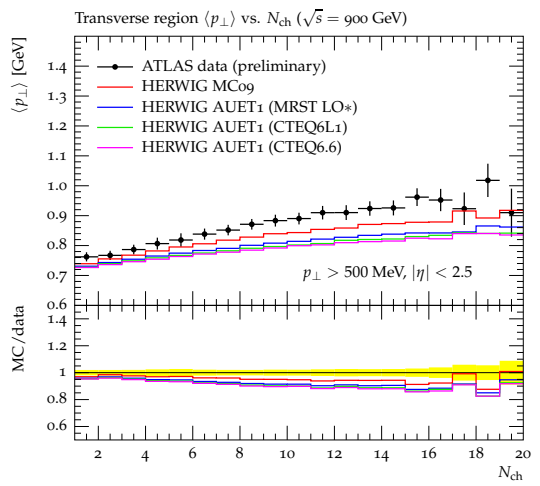

\section{$7 \mathrm{TeV}$}

Transverse region $\left\langle p_{\perp}\right\rangle$ vs. $N_{\text {ch }}(\sqrt{s}=7 \mathrm{TeV})$

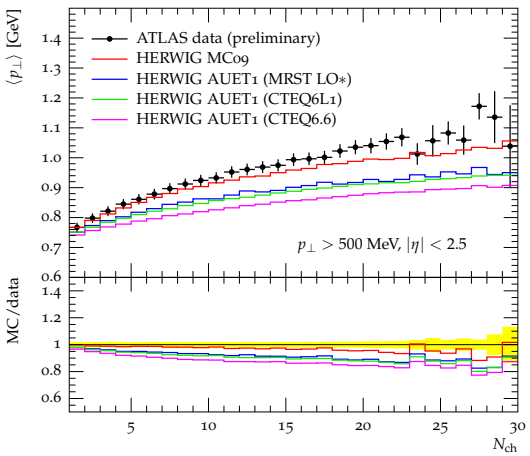




\section{Summary}

- PYTHIA AMBT1 and HERWIG+JIMMY AUET1 tunes from ATLAS give a good description of ATLAS soft QCD physics without severely compromising Tevatron agreement.

- Rough agreement of main MPI cutoff and energy-scaling exponent between PYTHIA/JIMMY, and between the pre-LHC and LHC tunes.

- Room for improvement:

- PYTHIA: final state shower and hadronisation not tuned to LEP data... improvements definitely possible. Also, better description of hadron collider jet shapes possible with recent PYTHIA versions.

- HERWIG: tuning to LEP not tried for a long time, can maybe use extra parameters for beam remnant fragmentation to better balance the momentum distribution among final-state particles.

- More generators: external work on Pythia 8, Herwig++ and Sherpa very promising: will be used in ATLAS. 